\title{
Assessment of a biofluid mechanics-based model for calculating portal pressure in canines
}

\author{
Jia-Yun Lin ${ }^{1 \dagger}$, Chi-Hao Zhang ${ }^{1 \dagger}$, Lei Zheng ${ }^{1 \dagger}$, Chen-Lu Song ${ }^{2 \dagger}$, Wen-Sheng Deng ${ }^{1}$, Yi-Ming Zhu', Li Zheng ${ }^{3}$, \\ Li-Zhong $\mathrm{Wu}^{4}$, Long-Ci Sun ${ }^{5^{*}}$ and Meng Luo ${ }^{1 *}$
}

\begin{abstract}
Background: Portal hypertension is a severe complication caused by various chronic liver diseases. The standard methods for detecting portal hypertension (hepatic venous pressure gradient and free portal pressure) are available in only a few hospitals due to their technical difficulty and invasiveness; thus, non-invasive measuring methods are needed. This study aimed to establish and assess a novel model to calculate free portal pressure based on biofluid mechanics.

Result: Comparison of each dog's virtual and actual free portal pressure showed that a biofluid mechanics-based model could accurately predict free portal pressure (mean difference: $-0.220,95 \% \mathrm{Cl}$ : -0.738 to 0.298 ; upper limit of agreement: 2.24, 95\% Cl: 1.34 to 3.14; lower limit of agreement: $-2.68,95 \%$ Cl: -3.58 to -1.78 ; intraclass correlation coefficient: 0.98 , 95\% Cl: 0.96 to 0.99; concordance correlation coefficient: 0.97, 95\% Cl: 0.93 to 0.99$)$ and had a high AUC (0.984, 95\% Cl: 0.834 to 1.000$)$, sensitivity $(92.3,95 \% \mathrm{Cl}: 64.0$ to 99.8$)$, specificity $(91.7,95 \% \mathrm{Cl}: 61.5$ to 99.8), positive likelihood ratio (11.1, $95 \%$ Cl: 1.7 to 72.8$)$, and low negative likelihood ratio (0.08, 95\% Cl: 0.01 to 0.6$)$ for detecting portal hypertension.

Conclusions: Our study suggests that the biofluid mechanics-based model was able to accurately predict free portal pressure and detect portal hypertension in canines. With further research and validation, this model might be applicable for calculating human portal pressure, detecting portal hypertensive patients, and evaluating disease progression and treatment efficacy.
\end{abstract}

Keywords: Biofluid mechanics, Non-invasive, Portal hypertension, Portal pressure

\section{Background}

Portal hypertension is known as a severe disease with a poor outcome. It can threaten people's lives if the gastroesophageal varices rupture and are not treated in time [1, 2]. Portal pressure measurement is of great importance, because it is an effective method for evaluating liver disease

\footnotetext{
*Correspondence: renjisunlongci@163.com; luosh9hospital@sina.com ${ }^{\dagger}$ Jia-Yun Lin, Chi-Hao Zhang, Lei Zheng and Chen-Lu Song contributed equally to this work.

${ }^{5}$ Department of Gastrointestinal Surgery, Renji Hospital, School of Medicine, Shanghai Jiao Tong University, No.160 Pujian Road, Shanghai 200127, China 'Department of General Surgery, Shanghai Ninth People's Hospital, School of Medicine, Shanghai Jiao Tong University, No. 639 Zhizaoju Road, Shanghai 200011, China

Full list of author information is available at the end of the article
}

progression [3]. However, the application of the hepatic venous pressure gradient (HVPG) and free portal pressure (FPP), the standard methods for detecting portal hypertension, are restricted due to technical difficulty and invasiveness [4-6]. Although there have been studies of noninvasive portal hypertension assessment methods, including clinical examination, ultrasound, elastography, CT and magnetic resonance imaging $[7,8]$, few were proven to be reliable. Recently, Yang [9-11] established threedimensional hepatic portal venous models from CT images to evaluate the severity of cirrhotic patients, which showed good performance; however, the models were neither introduced in detail nor validated in a large population.

(c) The Author(s). 2020 Open Access This article is licensed under a Creative Commons Attribution 4.0 International License, which permits use, sharing, adaptation, distribution and reproduction in any medium or format, as long as you give appropriate credit to the original author(s) and the source, provide a link to the Creative Commons licence, and indicate if changes were made. The images or other third party material in this article are included in the article's Creative Commons licence, unless indicated otherwise in a credit line to the material. If material is not included in the article's Creative Commons licence and your intended use is not permitted by statutory regulation or exceeds the permitted use, you will need to obtain permission directly from the copyright holder. To view a copy of this licence, visit http://creativecommons.org/licenses/by/4.0/ The Creative Commons Public Domain Dedication waiver (http://creativecommons.org/publicdomain/zero/1.0/) applies to the data made available in this article, unless otherwise stated in a credit line to the data. 
Therefore, an accurate and non-invasive portal pressure measuring method is needed and would be useful in the diagnosis and evaluation of portal hypertension.

Biofluid mechanics is the study of biological flow mechanisms and the inter-relationships with physiological and pathological processes using the fundamental principles of fluid mechanics [12]. Using biofluid mechanics, cardiologists succeeded in calculating fractional flow reserve, the standard assessment of the haemodynamics of coronary stenoses [13, 14]. This motivated us to establish a noninvasive method for accurately calculating FPP.

The aim of this study was to establish and assess a biofluid mechanics-based model for predicting FPP and detecting portal hypertension in canines.

\section{Results}

To validate canine portal hypertensive models, we focused on canine liver fibrogenesis (Fig. 1), liver function, FPP (Table 1), as well as blood vessel diameters (Table 2) and blood flow velocity (Table 3 ) of their portal venous system. We found that canines from $\mathrm{CCl}_{4}$-treated groups developed liver fibrogenesis, had lower blood flow velocity, wider blood vessel diameters, and higher liver function and FPP values as compared with those from a control group. These results proved the success of our canine portal hypertensive model.

We imported canine abdominal CT images into the IQQA-Liver system to produce a three-dimensional portal venous model, in which the portal vein and its main branches, including the left and right portal vein, the splenic vein, the superior mesenteric vein, and the inferior mesenteric vein, were visualized precisely. We used FLUENT software to divide the model (one case is shown in Fig. 2a), mesh the model surfaces into triangular surface grids and create the body meshes accordingly (one case is shown in Fig. 2b). Blood density and overall viscosity were used as the properties of the blood. The "pressure outlet boundary conditions" module was used for the portal vein and the "velocity inlet boundary conditions" module was used for each inlet and outlet branch. The blood flow velocity at the boundaries of each branch was calculated (formula 1-2). FLUENT software was used to solve the Navier-Stokes equations (formula 3-8), simulate the blood flow within the portal venous system (one case is shown in Fig. 3), and obtain the virtual free portal pressure (vFPP) (one case is shown in Fig. 4).

To assess the numeric correlation between vFPP and FPP, we performed Bland and Altman's limits of agreement analysis (Fig. 5a), the intraclass correlation coefficient (Fig. 5b), and Lin's concordance correlation coefficient (Fig. 5c) between FPP and vFPP. For Bland and Altman's limits of agreement analysis, the mean of the difference was -0.220 ( $95 \% \mathrm{CI}$ : -0.738 to 0.298 ); the upper limit of agreement was 2.24 (95\% CI: 1.34 to 3.14); the lower limit of agreement was -2.68 (95\% CI: -3.58 to -1.78$)$. The intraclass correlation coefficient was 0.98 (95\% CI: 0.96 to $0.99, P<0.0001)$. The concordance correlation coefficient was 0.97 (95\% CI: 0.93 to 0.99). These results showed that the vFPP model provided a good prediction of FPP.

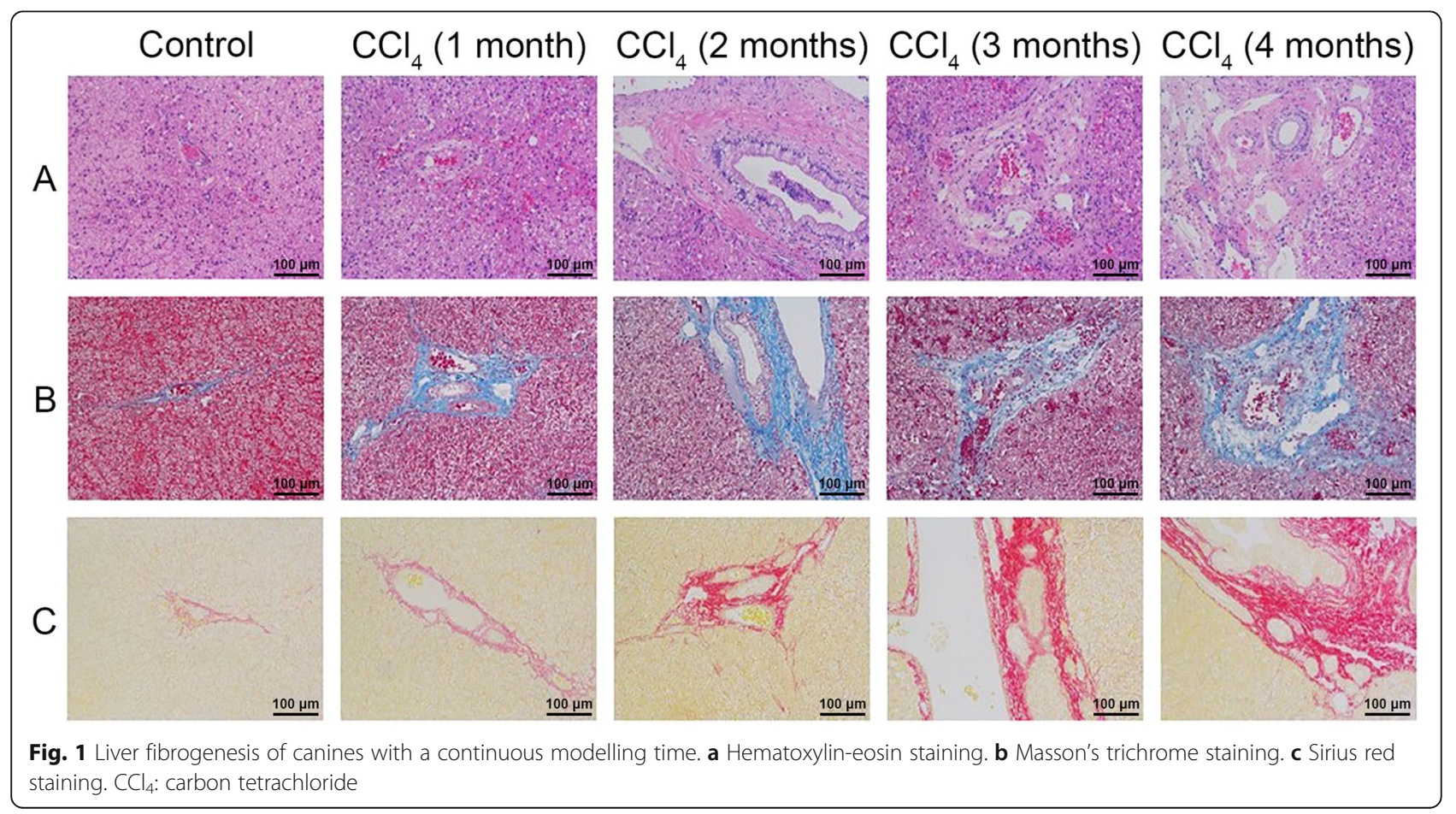


Table 1 Liver function and FPP of canines

\begin{tabular}{|c|c|c|c|c|c|}
\hline Parameters & Control & $\begin{array}{l}\mathrm{CCl}_{4} \\
\text { (1 month) }\end{array}$ & $\begin{array}{l}\mathrm{CCl}_{4} \\
\text { (2 months) }\end{array}$ & $\begin{array}{l}\mathrm{CCl}_{4} \\
\text { (3 months) }\end{array}$ & $\begin{array}{l}\mathrm{CCl}_{4} \\
\text { (4 months) }\end{array}$ \\
\hline Total bilirubin $(\mu \mathrm{mol} / \mathrm{L})$ & $2.2 \pm 0.9$ & $4.6 \pm 1.0^{*}$ & $6.5 \pm 0.7^{*}$ & $5.2 \pm 2.5$ & $7.3 \pm 0.8^{*}$ \\
\hline Direct bilirubin $(\mu \mathrm{mol} / \mathrm{L})$ & $0.5 \pm 0.3$ & $0.5 \pm 2.9$ & $2.3 \pm 0.3^{*}$ & $2.3 \pm 2.3$ & $3.4 \pm 1.3^{*}$ \\
\hline Indirect bilirubin $(\mu \mathrm{mol} / \mathrm{L})$ & $1.7 \pm 0.7$ & $4.0 \pm 0.9^{*}$ & $4.2 \pm 0.6^{*}$ & $3.0 \pm 0.3^{*}$ & $3.9 \pm 0.8^{*}$ \\
\hline Alanine aminotransferase (U/L) & $21 \pm 8$ & $81 \pm 47^{*}$ & $481 \pm 178^{*}$ & $78 \pm 46$ & $366 \pm 110^{*}$ \\
\hline Aspartate transaminase $(\mathrm{U} / \mathrm{L})$ & $28 \pm 9$ & $36 \pm 8$ & $50 \pm 20$ & $29 \pm 12$ & $58 \pm 13^{*}$ \\
\hline free portal pressure $(\mathrm{mmHg})$ & $6.6 \pm 1.1$ & $10.0 \pm 1.6^{*}$ & $13.6 \pm 1.5^{*}$ & $15.6 \pm 4.0^{*}$ & $19.2 \pm 1.9^{*}$ \\
\hline
\end{tabular}

Note. Values are presented as the $\mathrm{M} \pm \mathrm{SD}$; ${ }^{*}$ meaning $P<0.05$ vs. Control group; $\mathrm{CCl}_{4} \mathrm{Carbon}$ tetrachloride

To evaluate the performance of vFPP in the diagnostics of portal hypertension, we carried out receiver operating characteristic (ROC) curve analysis (Fig. 5d). The area under the ROC curve (AUC) was 0.984 (95\% CI: 0.834 to $1.000, P<0.0001)$. We selected $\mathrm{vFPP}=12$ $\mathrm{mmHg}$ as the criterion value. The sensitivity was 92.3 (95\% CI: 64.0 to 99.8); the specificity was 91.7 (95\% CI: 61.5 to 99.8 ); the positive likelihood ratio was 11.1 (95\% CI: 1.7 to 72.8 ); the negative likelihood ratio was 0.08 (95\% CI: 0.01 to 0.6).

All canines survived and had no important adverse events during the experiment.

\section{Discussion}

Portal hypertension is a life-threatening disease. Portal pressure measurement is of great importance because it is the standard method for detecting portal hypertension. Moreover, portal pressure strongly correlates with severe complications, such as hepatocellular carcinomas, gastrooesophageal variceal haemorrhaging, hepatic encephalopathy, and ascites [15]. In this study, we performed canine experiments to evaluate a biofluid mechanics-based model for calculating vFPP and detecting portal hypertension. Both FPP and HVPG are the standard methods for detecting portal hypertension $[1,2]$, however, we used only FPP as a reference because canines' FPP can be easily measured, and balloon-tipped catheters for canine HVPG measurements were unavailable due to the narrowness of the canine vessels.

The IQQA system, the FLUENT software, and the Navier-Stokes equations have been widely used to reconstruct blood vessels, simulate blood flow, and precisely calculate the haemodynamics [16-24]. In the portal venous system, due to the relatively tiny size of blood cells (compared with the size of the vessels) and the steadily fast blood flow, the blood within could be modelled as an incompressible Newtonian fluid; therefore, it is appropriate to apply the IQQA system, the FLUENT software and the Navier-Stokes equations to our study [25, 26]. Based on previous studies, we built the vFPP calculation model using IQQA and FLUENT software to reconstruct canine portal venous system, simulate the blood flow, and calculate the vFPP by the Navier-Stokes equations. After assessing the model by Bland and Altman's limits of agreement analysis, the intraclass correlation coefficient, Lin's concordance correlation coefficient and ROC curve analysis, we showed that this model was able to predict FPP and diagnose portal hypertension accurately.

Iranmanesh and Liu also established models for detecting portal hypertensive patients, which showed good diagnostic performance with high sensitivity and specificity $[27,28]$. Although their works were able to detect patients suffering from clinically significant portal hypertension (HVPG greater than $10 \mathrm{mmHg}$ ) well, their models were not suitable for mild or moderate portal

Table 2 Blood vessel diameters ( $\mathrm{mm}$ ) of canines' portal venous system

\begin{tabular}{|c|c|c|c|c|c|}
\hline$\overline{V e s s e l}(\mathrm{~mm})$ & Control & $\begin{array}{l}\mathrm{CCl}_{4} \\
\text { (1 month) }\end{array}$ & $\begin{array}{l}\mathrm{CCl}_{4} \\
\text { (2 months) }\end{array}$ & $\begin{array}{l}\mathrm{CCl}_{4} \\
\text { (3 months) }\end{array}$ & $\begin{array}{l}\mathrm{CCl}_{4} \\
\text { (4 months) }\end{array}$ \\
\hline Portal vein & $4.5 \pm 0.4$ & $7.2 \pm 0.6^{*}$ & $7.7 \pm 1.2^{*}$ & $8.2 \pm 1.0^{*}$ & $9.5 \pm 1.3^{*}$ \\
\hline Left portal vein & $3.4 \pm 0.3$ & $4.9 \pm 0.7^{*}$ & $5.9 \pm 0.9^{*}$ & $6.2 \pm 1.4^{*}$ & $7.6 \pm 1.2^{*}$ \\
\hline Right portal vein & $4.2 \pm 0.2$ & $4.9 \pm 0.4^{*}$ & $5.2 \pm 0.6^{*}$ & $5.8 \pm 0.6^{*}$ & $6.8 \pm 1.2^{*}$ \\
\hline Splenic vein & $1.8 \pm 0.2$ & $3.4 \pm 0.5^{*}$ & $4.0 \pm 0.5^{*}$ & $4.5 \pm 0.7^{*}$ & $4.9 \pm 0.8^{*}$ \\
\hline Superior mesenteric vein & $3.1 \pm 0.3$ & $4.1 \pm 0.2^{*}$ & $4.8 \pm 0.5^{*}$ & $5.8 \pm 0.7^{*}$ & $6.0 \pm 0.6^{*}$ \\
\hline Inferior mesenteric vein & $2.0 \pm 0.1$ & $2.4 \pm 0.3^{*}$ & $2.9 \pm 0.3^{*}$ & $3.5 \pm 0.4^{*}$ & $3.8 \pm 0.4^{*}$ \\
\hline
\end{tabular}

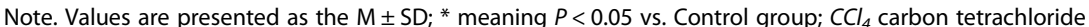


Table 3 Blood flow velocity $(\mathrm{cm} / \mathrm{s})$ of canines' portal venous system

\begin{tabular}{llllll}
\hline Vessel $(\mathrm{cm} / \mathrm{s})$ & Control & $\begin{array}{l}\mathrm{CCl}_{4} \\
(1 \mathrm{month})\end{array}$ & $\begin{array}{l}\mathrm{CCl}_{4} \\
(2 \text { months })\end{array}$ & $\begin{array}{l}\mathrm{CCl}_{4} \\
(3 \mathrm{months})\end{array}$ & $\begin{array}{l}\mathrm{CCl}_{4} \\
(4 \mathrm{months})\end{array}$ \\
\hline Portal vein & $33.8 \pm 8.7$ & $30.6 \pm 6.3$ & $24.8 \pm 3.7$ & $25.1 \pm 1.5$ & $11.4 \pm 2.3^{*}$ \\
Left portal vein & $22.6 \pm 4.4$ & $23.2 \pm 2.9$ & $13.7 \pm 3.4^{*}$ & $1.6^{*}$ \\
Right portal vein & $24.2 \pm 4.2$ & $25.4 \pm 1.3$ & $14.5 \pm 4.9$ & $11.7 \pm 2.1^{*}$ & $10.7 \pm 0.5^{*}$ \\
Splenic vein & $16.5 \pm 2.3$ & $16.5 \pm 2.0$ & $13.8 \pm 2.9$ & $18.5 \pm 6.5$ & $13.7 \pm 2.6^{*}$ \\
Superior mesenteric vein & $27.3 \pm 4.3$ & $27.5 \pm 4.5$ & $18.0 \pm 0.9^{*}$ & $17.7 \pm 2.3^{*}$ & $16.5 \pm 3.0^{*}$ \\
Inferior mesenteric vein & $15.0 \pm 1.6$ & $13.0 \pm 1.6$ & $10.7 \pm 1.8^{*}$ & $11.1^{*}$ & $10.3 \pm 0.5^{*}$
\end{tabular}

Note. Values are presented as the $\mathrm{M} \pm \mathrm{SD}$; ${ }^{*}$ meaning $P<0.05$ vs. Control group; $\mathrm{CCl}_{4} \mathrm{Carbon}$ tetrachloride

hypertensive patients whose HVPG were less than 10 mmHg. Therefore, we administered $\mathrm{CCl}_{4}$ to dogs for 1,2 , 3 or 4 months to represent different stages of portal hypertension. Our result showed that our model was suitable for not only severe but also mild portal hypertensive and even normal canine functionality. Moreover, our model was able to predict the vFPP value, which was another advantage over previous models. We used five time points $(0,1,2,3,4$ months), because there were few references reporting the adverse events for this method. We were concerned that some canines might be unable to survive 4 months of continuous injection of $\mathrm{CCl}_{4}$ and planned to terminate $\mathrm{CCl}_{4}$ injection if two dogs from the earliest group died. For example, if two dogs had lived for only 2 months during continuous injection of $\mathrm{CCl}_{4}$, then we would have terminated $\mathrm{CCl}_{4}$ injection at that time point; therefore, there would have been only three groups left: Control, $\mathrm{CCl}_{4}$ (1 month), and $\mathrm{CCl}_{4}$ (2 months). However, if we had designed only three time points $(0,2,4$ months) in this study, then in this situation, there would have been only two groups left: Control and $\mathrm{CCl}_{4}(2$ months), which would have been insufficient to reflect different stages of cirrhosis and portal hypertension.

Our non-invasive methods could calculate vFPP from CT, blood tests, and Doppler ultrasound results. Similarly, we could use these methods to simulate the blood flow of the inferior vena cava and hepatic veins, and calculate the virtual hepatic venous pressure (vHVP) and virtual HVPG (the difference between vFPP and vHVP). With further research and validation, this model might be applicable for calculating human vFPP and virtual HVPG, detecting portal hypertensive patients, and evaluating disease progression and treatment efficacy.

There are some limitations in this study. Firstly, although we demonstrated a high correlation between vFPP and FPP, this result was acquired from canines, not humans. We chose beagles as experimental animals because they have a moderate body size and a digestive system similar to humans. It is also easy to perform examinations on them. In addition, the methods and results of this study might be applied to humans more appropriately. After this study, we applied this model to several portal hypertensive patients who underwent portosystemic shunts or splenectomy with periesophagogastric devascularisation and found similar results (unpublished observations). We are currently carrying out a prospective, randomised, non-controlled, multicentre trial (trial registration number: NCT03470389) to further validate this model in humans [29]. Secondly, the intra-abdominal pressure might have changed after

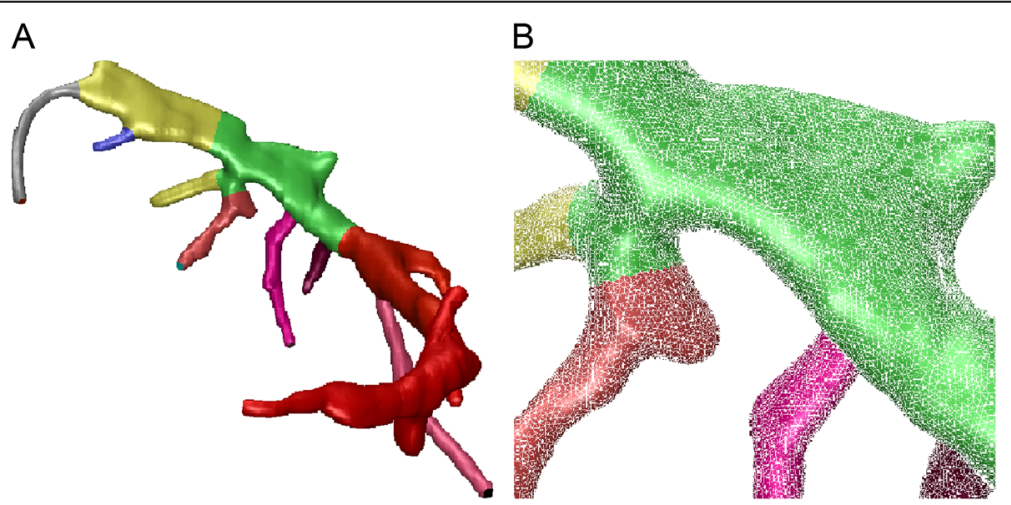

Fig. 2 a The simulation model of the portal venous system, different colours representing different parts. $\mathbf{b}$ The body meshes of the simulation model 


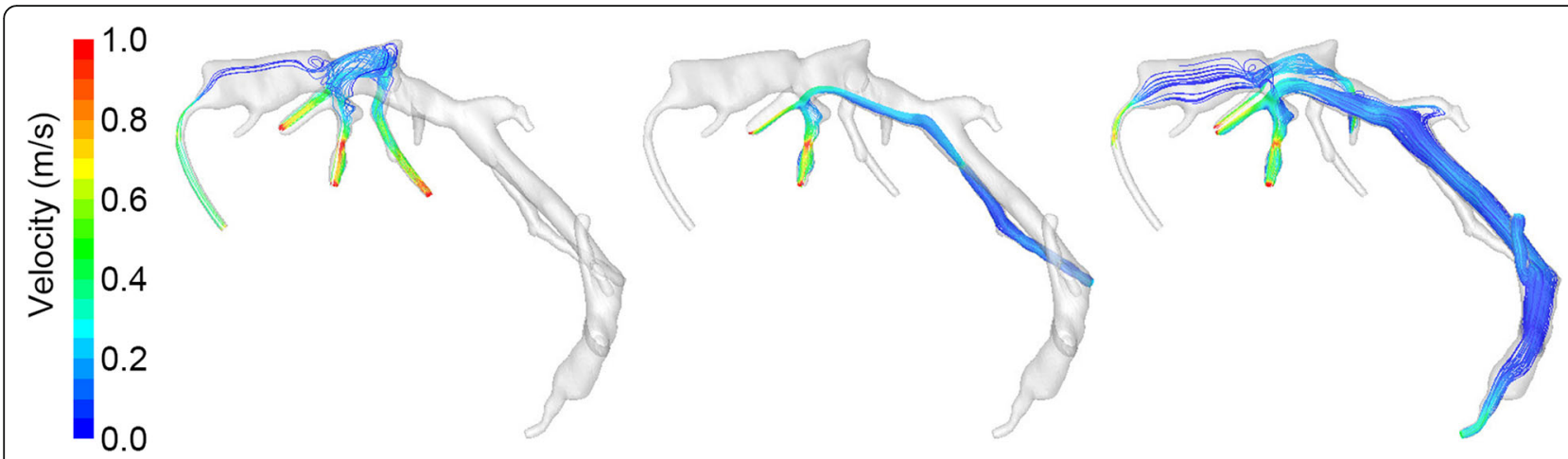

Fig. 3 The blood flow simulation of the portal venous system

general anaesthesia and abdominal incision, which might have influenced the FPP. Thirdly, the accuracy of the time-intensity curves of the Doppler ultrasound might have been affected by respiratory motion artefacts; this problem was partly alleviated by limiting the breathing extent of the canines.

\section{Conclusion}

The non-invasive and biofluid mechanics-based model could accurately predict FPP and had high sensitivity and specificity for detecting portal hypertension in canines. With further research and validation, this model might be applicable for calculating human FPP, detecting patients with portal hypertension, and evaluating disease progressions and treatment efficacies.

\section{Methods}

\section{Animal model}

The canines used in this study were purchased from the laboratory animal department of Shanghai Jiagan Biotechnology Co., Ltd. as experimental animals. The study protocol was reviewed and approved by the Animal Care and Use Committee of Shanghai Ninth People's Hospital,
School of Medicine, Shanghai Jiao Tong University. All procedures were conducted according to the Animal Experimentation Guidelines of Shanghai Jiao Tong University. The study was performed on 25 adult male beagles $(10.5$ to $12.5 \mathrm{~kg})$, which were caged with constant temperature $\left(25^{\circ} \mathrm{C}\right)$, humidity $(60 \pm 10 \%)$, and circadianrhythmic lighting in the laboratory animal department of Shanghai Jiagan Biotechnology Co., Ltd. To reflect different stages of cirrhosis and portal hypertension, canine portal hypertensive models were induced by continuous subcutaneous injection of $\mathrm{CCl}_{4}$. The 25 dogs were divided randomly into five 5-member groups: Control, $\mathrm{CCl}_{4}(1$ month), $\mathrm{CCl}_{4}$ (2 months), $\mathrm{CCl}_{4}$ (3 months), and $\mathrm{CCl}_{4}$ (4 months). Randomisation was based on a computergenerated random digits table. Each dog's group was blind to the researchers responsible for histological staining, laboratory tests, Doppler ultrasound, CT, FPP measurement, and vFPP computation in order to prevent biases. The 20 dogs in the $\mathrm{CCl}_{4}$-treated groups began receiving $\mathrm{CCl}_{4}$ administration 1, 2, 3 or 4 months before the end of the study, so that all dogs reached the end of the study simultaneously. $\mathrm{CCl}_{4}$ was dissolved in olive oil $\left(60 \% \mathrm{CCl}_{4}\right.$ and $40 \%$ olive oil) and injected subcutaneously in the

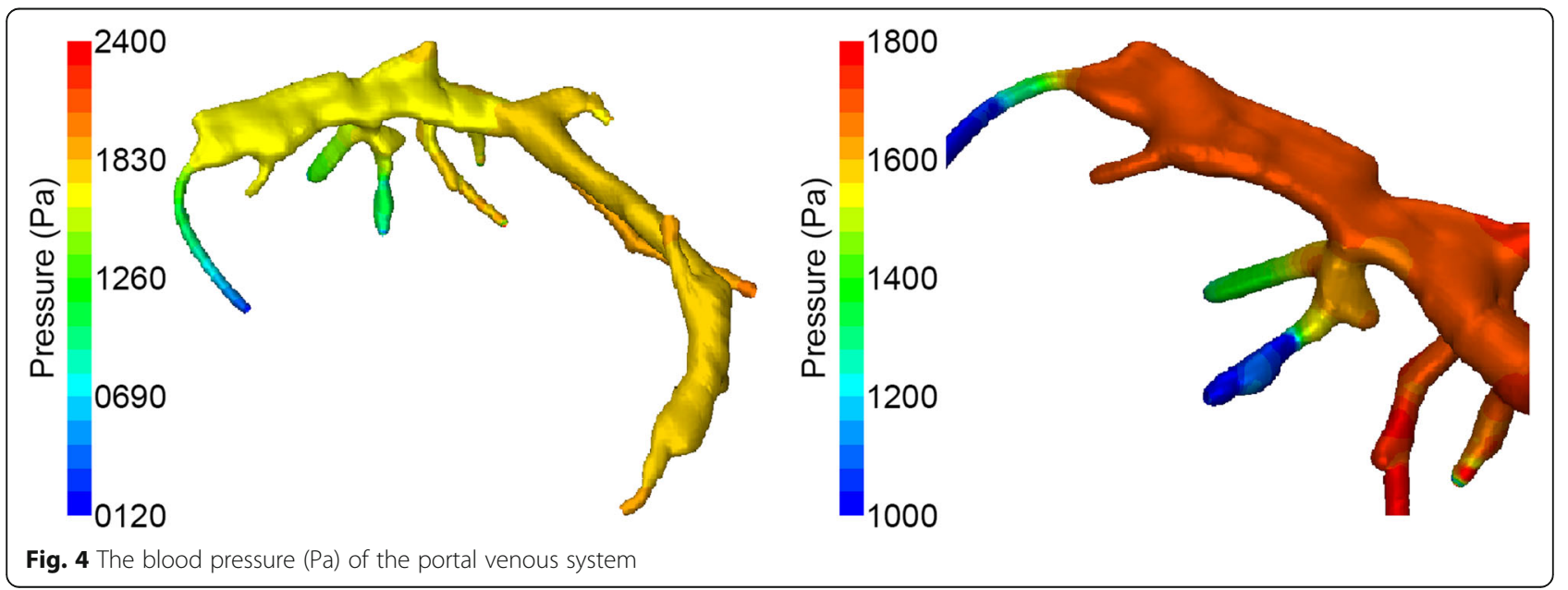



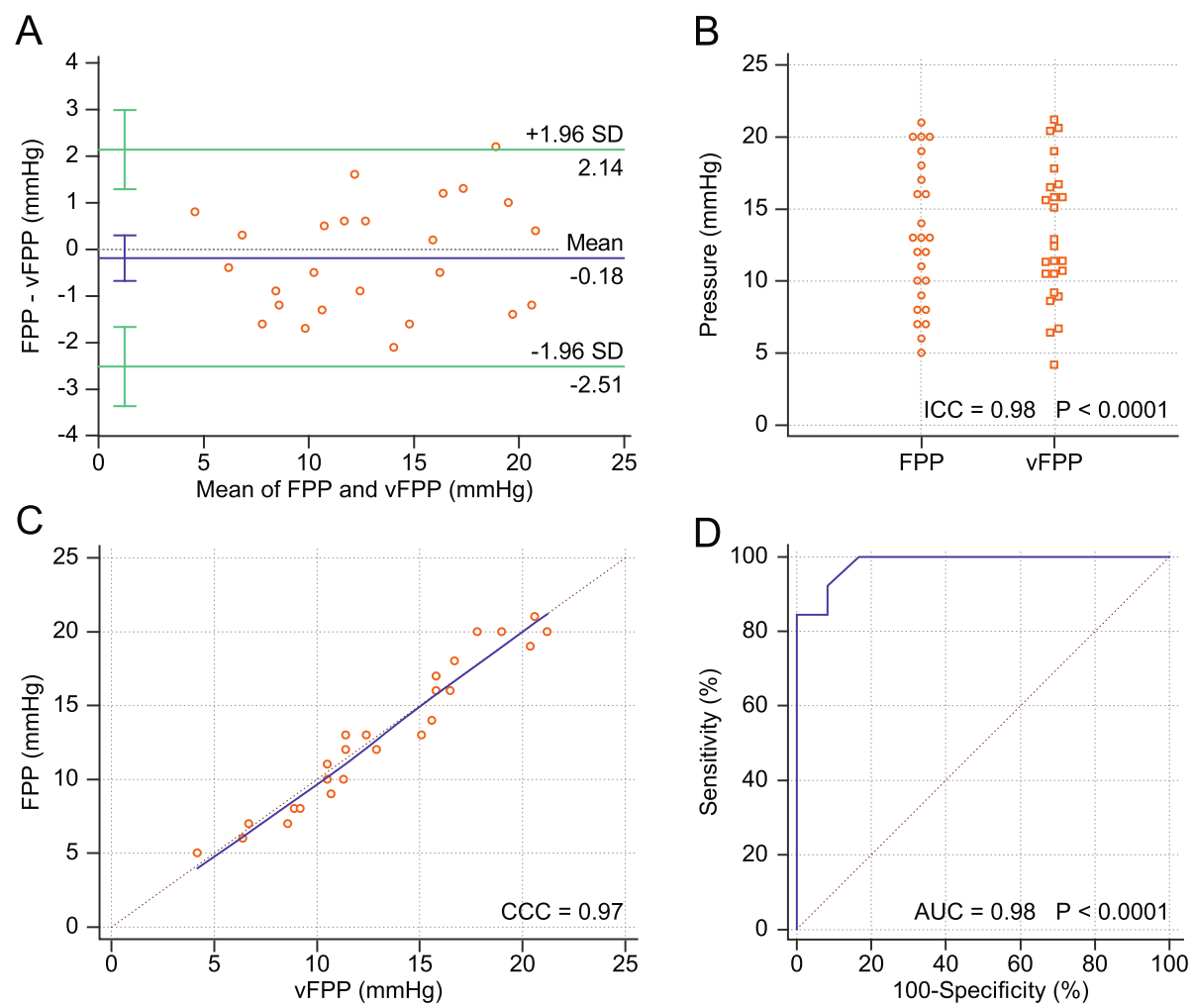

Fig. 5 The numeric correlation between VFPP and FPP and the diagnostic performance of VFPP. a Bland and Altman's limits of agreement analysis. b Intraclass correlation coefficient (ICC). c Lin's concordance correlation coefficient (CCC). $\mathbf{d}$ Receiver Operating Characteristic (ROC) curve and Area under the ROC curve (AUC) of VFPP. FPP: free portal pressure; VFPP: virtual free portal pressure

dorsal area of the canines. This administration was repeated every 10 days at a dose of 1.0 to $1.3 \mathrm{ml} / \mathrm{kg}$. Histological staining, laboratory tests, Doppler ultrasound, CT, FPP measurement, and vFPP computation were performed at the end of the study. All canines survived and continued to live in the laboratory animal department of Shanghai Jiagan Biotechnology Co., Ltd. after the study.

\section{Histological staining}

Hepatic tissue was taken and fixed in 10\% formalin and embedded in paraffin. The paraffin-embedded tissue was sectioned at $5 \mu \mathrm{m}$ and then placed on slides, deparaffinized in xylene, hydrated in decreasing concentrations of ethanol, and washed in water. After hematoxylin-eosin staining, Masson's trichrome staining, and Sirius red staining, the sections were examined under a microscope.

\section{Laboratory test}

Each dog's peripheral blood samples were taken from the small saphenous vein of the hind leg for blood viscosity and liver function tests. The blood density test was performed by weighing 1 millilitre of blood using an electronic balance. The blood density measurements were repeated at least three times and then averaged.

\section{Doppler ultrasound}

Each canine underwent an abdominal Doppler ultrasound scan after an overnight fast and a venous injection of pentobarbital sodium $(30 \mathrm{mg} / \mathrm{kg})$ in accordance with previously published methods [30]. Each canine was in dorsal recumbency throughout the scan. The ultrasound specialists measured the inner diameters and the maximum blood flow velocity of the portal vein and its main branches, including the right branch of the portal vein, the left branch of the portal vein, the portal vein, the splenic vein, the superior mesenteric vein, and the inferior mesenteric vein. The $3-5 \mathrm{MHz}$ Doppler ultrasound probes were used and the insonation angles were between $45^{\circ}$ and $65^{\circ}$. Each measurement was repeated twice; both intra-observer variability and inter-observer variability were less than $10 \%$.

\section{Computed tomography}

Each dog was fixed in the supine position and underwent an abdominal contrast-enhanced CT after an overnight fast and a venous injection of pentobarbital sodium (30 $\mathrm{mg} / \mathrm{kg}$ ) in accordance with previously published methods $[31,32]$. A non-ionic iodinated contrast agent $(600 \mathrm{mg}$ of iodine per kilogram of body weight, $300 \mathrm{mg}$ of iodine per $\mathrm{ml}, 5 \mathrm{ml}$ per second) was injected. The arterial phase 
imaging began $10 \mathrm{~s}$ after the beginning of the intravenous contrast injection, and portal phase imaging began 30 to $40 \mathrm{~s}$ after the beginning of the intravenous contrast injection.

\section{FPP measurement}

Each canine's FPP was measured after general anaesthesia. An abdominal midline incision was made, exposing the right gastroepiploic vein, and a pressure sensor-connected catheter was inserted into the portal vein through the right gastroepiploic vein. The FPP was recorded by a physiological signal acquisition system. The right atrium pressure was defined as the zero reference point.

\section{vFPP computation}

The simulation model of the canine portal venous system, which was created from canine CT images by the IQQA-Liver system version 2.0 (EDDA Technology, Inc., USA), was imported into the Fluent software version 6.3 (ANSYS, Inc., USA). The model was divided into different parts, with each inlet and outlet branch identified as a separate part. The model surface was then meshed into $0.2-1.0 \mathrm{~mm}$ triangular surface grids and the body meshes were created accordingly. The laminar viscous model was used. The material type was set to fluid, blood density and overall viscosity were used as the properties of the fluid. The pressure outlet boundary conditions module was used for the portal vein, with the following parameters: backflow reference frame: absolute; gauge pressure: 0 ; backflow direction specification method: normal to boundary; radial equilibrium pressure distribution: disabled; average pressure specification: disabled; target mass flow rate: disabled. The velocity inlet boundary conditions module was used for each inlet and outlet branch, with the following parameters: velocity specification method: magnitude, normal to boundary; reference frame: absolute; supersonic/initial gauge pressure: 0 . The velocity magnitude value was set to the blood flow velocity at the boundaries of each branch, which was calculated according to the inner diameter, blood flow velocity and direction measured by Doppler ultrasound, the inner diameter at the boundaries obtained from the simulation model, and the principle of mass conservation. The equations are as follows:

$$
\begin{aligned}
& \mathrm{Q}=\mathrm{Av}=\frac{1}{4} \pi \mathrm{d}_{\mathrm{b}}^{2} \mathrm{v}_{\mathrm{b}}=\frac{1}{4} \pi \mathrm{d}_{\mathrm{us}}^{2} \mathrm{v}_{\mathrm{us}} \\
& \mathrm{v}_{\mathrm{b}}=\frac{\mathrm{d}_{\mathrm{us}}^{2}}{\mathrm{~d}_{\mathrm{b}}^{2}} \mathrm{v}_{\mathrm{us}}
\end{aligned}
$$

Q: volume flow rate; A: cross-sectional area; v: velocity; $\mathrm{d}_{\mathrm{b}}$ : inner diameter at the boundaries; $\mathrm{v}_{\mathrm{b}}$ : velocity at the boundaries; $\mathrm{d}_{\mathrm{us}}$ : inner diameter measured by Doppler ultrasound; $\mathrm{v}_{\mathrm{us}}$ : velocity measured by Doppler ultrasound.
According to Doppler ultrasound images (one case is shown in Fig. 6), the mean blood flow velocity approximately equals 0.7 times the maximum blood flow velocity. Since the blood within the portal venous system can be assumed to be an incompressible Newtonian fluid, blood flow was modelled by the Navier-Stokes equations as follows:

The mass conservation equation:

$$
\frac{\partial \rho}{\partial t}=\nabla \cdot(\rho \vec{v})=S_{m}
$$

$\rho$ : density; t: time; $S_{m}$ : mass added to the continuous phase.

For axisymmetric geometries, the mass conservation equation can be written as follows:

$$
\frac{\partial \rho}{\partial t}+\frac{\partial}{\partial x}\left(\rho v_{x}\right)+\frac{\partial}{\partial r}\left(\rho v_{r}\right)+\frac{\rho v_{r}}{r}=S_{m}
$$

$\mathrm{x}$ : axial coordinate; $\mathrm{r}$ : radial coordinate; $\mathrm{v}_{\mathrm{x}}$ : axial velocity; $\mathrm{v}_{\mathrm{r}}$ : radial velocity.

The momentum conservation equation:

$$
\frac{\partial}{\partial \mathrm{t}}(\rho \overrightarrow{\mathrm{v}})+\nabla \cdot(\rho \overrightarrow{\mathrm{v}} \overrightarrow{\mathrm{v}})=-\nabla \mathrm{p}+\nabla \cdot(\overline{\overline{\mathrm{\tau}}})+\rho \overrightarrow{\mathrm{g}}+\overrightarrow{\mathrm{F}}
$$

p: static pressure; $\overline{\bar{\tau}}$ : stress tensor; $\mathrm{p} \overrightarrow{\mathrm{g}}$ : gravitational body force; $\overrightarrow{\mathrm{F}}$ : external body force.

$\overline{\overline{\mathrm{\tau}}}$, the stress tensor, can be written as follows:

$$
\overline{\overline{\mathrm{\tau}}}=\mu\left[\left(\nabla \overrightarrow{\mathrm{v}}+\nabla \overrightarrow{\mathrm{v}}^{\mathrm{T}}\right)-\frac{2}{3} \nabla \cdot \overrightarrow{\mathrm{v}} \mathrm{I}\right]
$$

$\mu$ : molecular viscosity; I: unit tensor; T: temperature. The energy conservation equation:

$$
\frac{\partial}{\partial t}(\rho E)+\nabla \cdot(\vec{v}(\rho E+p))=\nabla \cdot\left(k_{\text {eff }} \nabla T-\Sigma_{j} h_{j} \vec{j}_{j}+\left(\overline{\bar{e}}_{\text {eff }} \cdot \vec{v}\right)\right)+S_{h}
$$

E: total energy of fluid; $k_{\text {eff: }}$ effective conductive coefficient; h: enthalpy; J: diffusion flux; $\mathrm{S}_{\mathrm{h}}$ : volumetric heat sources.

E, the total energy of fluid, can be written as follows:

$$
E=h-\frac{p}{\rho}+\frac{v^{2}}{2}
$$

The FLUENT software was used to solve the NavierStokes equations, simulate the blood flow within the portal venous system, and obtain the pressure on each volume grid. The vFPP equals the pressure at the centre of the portal vein. 


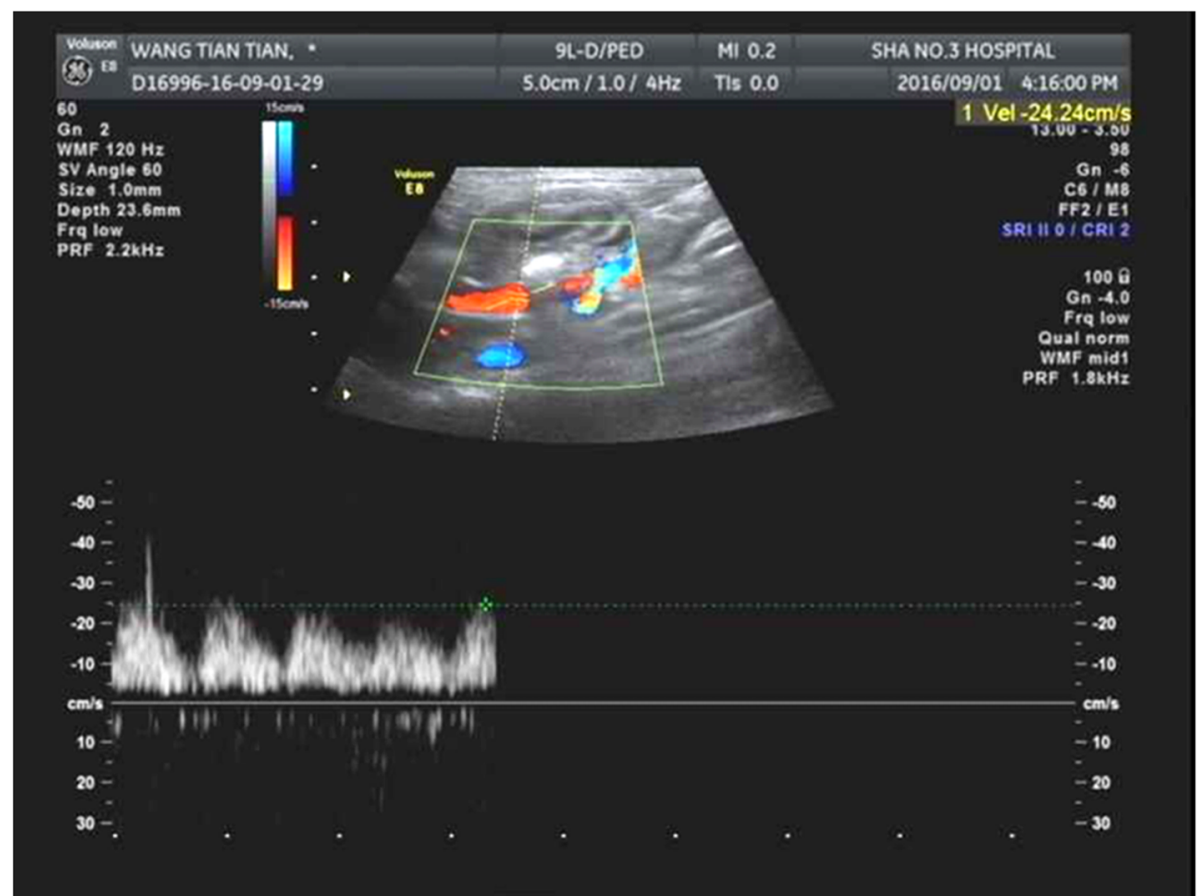

Fig. 6 The Doppler ultrasound image of the portal venous system

\section{Statistical analysis}

Continuous variables were checked for normal distribution and summarized by either mean \pm standard deviation or median and inter-quartile range, as appropriate. Comparison of continuous variables were performed using Student's t-test or analysis of variance for normally distributed variables and the Mann-Whitney $U$ test or the Kruskal-Wallis test for non-normally distributed variables as appropriate. The numeric correlation between FPP and vFPP was analysed by Bland and Altman's limits of agreement analysis [33]. Bias was defined as the mean of the difference between FPP and vFPP. Upper and lower limits of agreement were defined as mean difference \pm 1.96 standard deviation of the difference. The numeric correlation between FPP and vFPP was also analysed by the intraclass correlation coefficient and Lin's concordance correlation coefficient. The diagnostic accuracy of vFPP for detecting portal hypertension (FPP greater than $12 \mathrm{mmHg}$ ) was assessed by ROC curve analysis, the sensitivity, the specificity, and the likelihood ratio. All tests of significance were at the 5\% significance level. Analyses were performed using SPSS version 24.0 (IBM, USA) and MedCalc Statistical Software version 18.11 (MedCalc Software bvba, Belgium).

\section{Abbreviations}

CT: Computed tomography; Cl: Confidence interval; HVPG: Hepatic venous pressure gradient; FPP: Free portal pressure; $\mathrm{CCl}_{4}$ : Carbon tetrachloride; ROC: Receiver operating characteristic; AUC: Area under the ROC curve: ICC: Intraclass correlation coefficient; CCC: Concordance correlation coefficient; vFPP: Virtual free portal pressure; vHVP: Virtual hepatic venous pressure

\section{Acknowledgements}

The authors thank Xiao-Yu Yang for his assistance in biofluid mechanics.

\section{Authors' contributions}

$M L$ and L-CS conceived the study. J-YL and LZ1 were responsible for FPP measurement. C-HZ was responsible for VFPP computation. C-LS was responsible for statistical analysis. W-SD was responsible for histological staining. YMZ was responsible for laboratory test. LZ2 was responsible for Doppler ultrasound. L-ZW was responsible for CT. J-YL, C-HZ, LZ1 and C-LS drafted the protocol manuscript. All authors contributed to the design of the study and critically revised the successive versions of the manuscript and approved the final version.

\section{Funding}

This study was financially supported by the Clinical Research Program of Ninth People's Hospital, Shanghai Jiao Tong University School of Medicine (JYLJ021), Joint Project of Key Diseases of Shanghai Municipal Health Bureau (2014ZYJB0202) and National Natural Science Foundation of China (81770599, 81970526). The funders were not involved in the design of the study, the collection, analysis, interpretation of data or the writing of the manuscript.

\section{Availability of data and materials}

The datasets used and/or analysed during the current study are available from the corresponding author on reasonable request.

\section{Ethics approval and consent to participate}

The canines used in this study were purchased from the laboratory animal department of Shanghai Jiagan Biotechnology Co., Ltd. as experimental animals. The study protocol was reviewed and approved by the Animal Care and Use Committee of Shanghai Ninth People's Hospital, School of Medicine, Shanghai Jiao Tong University. All procedures were conducted according to the Animal Experimentation Guidelines of Shanghai Jiao Tong University. 


\section{Consent for publication}

Not applicable.

\section{Competing interests}

The authors declare that they have no competing interests.

\section{Author details}

'Department of General Surgery, Shanghai Ninth People's Hospital, School of Medicine, Shanghai Jiao Tong University, No. 639 Zhizaoju Road, Shanghai 200011 , China. ${ }^{2}$ Department of Plastic Surgery, Shanghai Ninth People's Hospital, School of Medicine, Shanghai Jiao Tong University, No. 639 Zhizaoju Road, Shanghai 200011, China. ${ }^{3}$ Department of Ultrasound, Shanghai Baoshan Integrated Traditional Chinese and Western Medicine Hospital, No. 181 Youyi Road, Shanghai 201900, China. ${ }^{4}$ Department of Radiation, Shanghai Ninth People's Hospital, School of Medicine, Shanghai Jiao Tong University, No. 639 Zhizaoju Road, Shanghai 200011, China. ${ }^{5}$ Department of Gastrointestinal Surgery, Renji Hospital, School of Medicine, Shanghai Jiao Tong University, No.160 Pujian Road, Shanghai 200127, China.

Received: 17 December 2019 Accepted: 14 July 2020

Published online: 26 August 2020

\section{References}

1. de Franchis R, Baveno VIF. Expanding consensus in portal hypertension: report of the Baveno $\mathrm{VI}$ consensus workshop: stratifying risk and individualizing care for portal hypertension. J Hepatol. 2015;63(3):743-52.

2. Tsochatzis EA, Bosch J, Burroughs AK. Liver cirrhosis. Lancet. 2014;383(9930): 1749-61.

3. Halpern EJ. Science to practice: noninvasive assessment of portal hypertension--can US aid in the prediction of portal pressure and monitoring of therapy? Radiology. 2006;240(2):309-10.

4. Bosch J, Abraldes JG, Berzigotti A, Garcia-Pagan JC. The clinical use of HVPG measurements in chronic liver disease. Nat Rev Gastroenterol Hepatol. 2009; 6(10):573-82.

5. de Franchis R, Dell'Era A. Invasive and noninvasive methods to diagnose portal hypertension and esophageal varices. Clinics in liver disease. 2014; 18(2):293-302.

6. Singal AK, Ahmad M, Soloway RD. Duplex Doppler ultrasound examination of the portal venous system: an emerging novel technique for the estimation of portal vein pressure. Dig Dis Sci. 2010;55(5):1230-40.

7. El-Saadany M, Jalil S, Irisawa A, Shibukawa G, Ohira H, Bhutani MS. EUS for portal hypertension: a comprehensive and critical appraisal of clinical and experimental indications. Endoscopy. 2008:40(8):690-6.

8. Qi X, Berzigotti A, Cardenas A, Sarin SK. Emerging non-invasive approaches for diagnosis and monitoring of portal hypertension. Lancet Gastroenterol Hepatol. 2018;3(10):708-19.

9. Qi X, Zhou F, Lv H, Chen H, Xu W, Xing S, Wang F, Yang C. A novel noninvasive assessment of hepatic venous pressure gradient and portal pressure computed from computed tomography angiography. Arch Med Sci. 2014;10(5):1052-4.

10. Qi X, Xu M, Li Z, Yang C. Virtual portal pressure from anatomic $C$ angiography. J Hepatol. 2014;61(1):180-1.

11. Qi X, Li Z, Huang J, Zhu Y, Liu H, Zhou F, Liu C, Xiao C, Dong J, Zhao Y, et al. Virtual portal pressure gradient from anatomic $C T$ angiography. Gut. 2015;64(6):1004-5

12. Elad D, Bluestein D. Biofluid mechanics: innovations and challenges. J Biomech. 2013;46(2):207.

13. Taylor CA, Fonte TA, Min JK. Computational fluid dynamics applied to cardiac computed tomography for noninvasive quantification of fractional flow reserve: scientific basis. J Am Coll Cardiol. 2013:61(22):2233-41.

14. Koo BK, Erglis A, Doh JH, Daniels DV, Jegere S, Kim HS, Dunning A, DeFrance T, Lansky A, Leipsic J, et al. Diagnosis of ischemia-causing coronary stenoses by noninvasive fractional flow reserve computed from coronary computed tomographic angiograms. Results from the prospective multicenter DISCOVER-FLOW (diagnosis of ischemia-causing Stenoses obtained via noninvasive fractional flow reserve) study. J Am Coll Cardiol. 2011:58(19):1989-97.

15. Tripathi D, Stanley AJ, Hayes PC, Patch D, Millson C, Mehrzad H, Austin A, Ferguson JW, Olliff SP, Hudson M, et al. U.K. quidelines on the management of variceal haemorrhage in cirrhotic patients. Gut. 2015;64(11):1680-704.
16. Xiang C, Chen Y, Shao M, Li C, Huang X, Gong L, Li A, Duan W, Zhang A, Dong J. Three-dimensional quantitative evaluation of the segmental functional Reserve in the Cirrhotic Liver Using Multi-Modality Imaging. Medicine (Baltimore). 2016;95(9):e2719.

17. Ji GW, Zhu FP, Wang K, Jiao CY, Shao ZC, Li XC. Clinical implications of biliary confluence pattern for bismuth-Corlette type IV Hilar Cholangiocarcinoma applied to Hemihepatectomy. J Gastrointest Surg. 2017:21(4):666-75.

18. He YB, Bai L, Jiang $Y$, Ji XW, Tai QW, Zhao JM, Zhang JH, Liu WY, Wen $H$. Application of a three-dimensional reconstruction technique in liver autotransplantation for end-stage hepatic alveolar Echinococcosis. J Gastrointest Surg. 2015;19(8):1457-65

19. He YB, Bai L, Aji T, Jiang Y, Zhao JM, Zhang JH, Shao YM, Liu WY, Wen $H$. Application of 3D reconstruction for surgical treatment of hepatic alveolar echinococcosis. World J Gastroenterol. 2015;21(35):10200-7.

20. Goubergrits L, Mevert R, Yevtushenko P, Schaller J, Kertzscher U, Meier S, Schubert S, Riesenkampff E, Kuehne T. The impact of MRI-based inflow for the hemodynamic evaluation of aortic coarctation. Ann Biomed Eng. 2013; 41(12):2575-87.

21. Goubergrits L, Riesenkampff E, Yevtushenko P, Schaller J, Kertzscher U, Berger F, Kuehne T. Is MRI-based CFD able to improve clinical treatment of coarctations of aorta? Ann Biomed Eng. 2015:43(1):168-76.

22. Ai L, Yu H, Dai W, Hale SL, Kloner RA, Hsiai TK. Real-time intravascular shear stress in the rabbit abdominal aorta. IEEE Trans Biomed Eng. 2009:56(6): 1755-64.

23. Liu J, Yan Z, Pu Y, Shiu WS, Wu J, Chen R, Leng X, Qin H, Liu X, Jia B, et al. Functional assessment of cerebral artery stenosis: a pilot study based on computational fluid dynamics. J Cereb Blood Flow Metab. 2017;37(7):256776.

24. Miraucourt O, Salmon S, Szopos M, Thiriet M. Blood flow in the cerebral venous system: modeling and simulation. Comput Methods Biomech Biomed Engin. 2017;20(5):471-82

25. Moon JY, Suh DC, Lee YS, Kim YW, Lee JS. Considerations of blood properties, outlet boundary conditions and energy loss approaches in computational fluid dynamics modeling. Neurointervention. 2014;9(1):1-8.

26. Tura A, Sarti A, Gaens T, Lamberti C. Regularization of blood motion fields by modified Navier-stokes equations. Med Eng Phys. 1999;21(1):27-36.

27. Iranmanesh P, Vazquez O, Terraz S, Majno P, Spahr L, Poncet A, Morel P, Mentha G, Toso C. Accurate computed tomography-based portal pressure assessment in patients with hepatocellular carcinoma. J Hepatol. 2014;60(5): 969-74.

28. Liu F, Ning Z, Liu Y, Liu D, Tian J, Luo H, An W, Huang Y, Zou J, Liu C, et al. Development and validation of a radiomics signature for clinically significant portal hypertension in cirrhosis (CHESS1701): a prospective multicenter study. EBioMedicine. 2018;36:151-8.

29. Lin JY, Zhang CH, Zheng L, Li HJ, Zhu YM, Fan X, Li F, Xia Y, Huang MZ, Yang $\mathrm{SH}$, et al. Establishment and assessment of the hepatic venous pressure gradient using biofluid mechanics (HVPGBFM): protocol for a prospective, randomised, non-controlled, multicentre study. BMJ Open. 2019;9(12):e028518.

30. Szatmari V, Sotonyi P, Voros K. Normal duplex Doppler waveforms of major abdominal blood vessels in dogs: a review. Vet Radiol Ultrasound. 2001; 42(2):93-107.

31. Ohlerth S, Scharf G. Computed tomography in small animals--basic principles and state of the art applications. Vet J. 2007;173(2):254-71.

32. Zwingenberger AL, Schwarz T. Dual-phase CT angiography of the normal canine portal and hepatic vasculature. Vet Radiol Ultrasound. 2004;45(2): 117-24.

33. Bland JM, Altman DG. Statistical methods for assessing agreement between two methods of clinical measurement. Lancet. 1986;1(8476):307-10.

\section{Publisher's Note}

Springer Nature remains neutral with regard to jurisdictional claims in published maps and institutional affiliations. 